\title{
Rupture of an Idiopathic Aneurysm of the Inferior Pancreaticoduodenal Artery Leading to an Intra-Abdominal Bleeding
}

\author{
Riham Imam a, Harbi Khalayleh b,*, Deeb Khoury b, Guy Lin b, Ashraf Imam b \\ a Department of General Surgery, Hadassah-Hebrew University Medical Center, Jerusalem, Israel \\ ${ }^{b}$ Department of Surgery, Kaplan Medical Center, Rehovot and The Hebrew University School of Medicine, Jerusalem, Israel
}

\section{Article history:}

Received: July 13, 2019

Revised: October 25, 2019

Accepted: November 12, 2019

\section{${ }^{*}$ Corresponding Author:}

Harbi Khalayleh

Department of Surgery, Kaplan Medical

Center, Rehovot and The Hebrew University

School of Medicine, Jerusalem, Israel

E-mail: kraceve30@hotmail.com

\section{ORCID}

Riham Imam

https://orcid.org/0000-0002-9841-2278

Harbi Khalayleh

https://orcid.org/0000-0002-6706-3837

Deeb Khoury

https://orcid.org/0000-0001-7830-1450

Ashraf Imam

https://orcid.org/0000-0003-0172-3844

\section{Introduction}

Pancreaticoduodenal artery (PDA) aneurysms are very rare [1] but potentially fatal [2]. Depending on the etiology of the aneurysm, it can be defined as a true or false aneurysm. True aneurysms of the PDA are more common, and are usually due to atherosclerosis or celiac stenosis [3]. Whilst a pseudoaneurysm, a false aneurysm, is caused by a previous attack of pancreatitis whether acute or chronic, or through trauma or iatrogenic reasons after surgery [4].

In this report, a case of spontaneous rupture of a PDA aneurysm in a patient with no significant previous medical history is presented.

\section{Case Report}

A 59-year old man with no previous medical history (other than smoking), presented to the Emergency Department with hemorrhagic shock, with no history of trauma, abdominal pain, or signs of gastrointestinal bleeding.

After resuscitation of the patient with crystalloid fluids and blood products for stabilization, an abdominal computed tomography angiography was performed. It showed a hematoma between the duodenum and the pancreas, with an active extravasation of contrast (Figures 1 and 2). There were no signs of pancreatitis on the computed tomography scan.

On admission the patient's hemoglobin (HGB) level was $10 \mathrm{~g} / \mathrm{dL}$; whilst baseline HGB was $14 \mathrm{~g} / \mathrm{dL}$, and the patient was acidotic with a PH of 6.9 , with a creatinine level of $1.63 \mathrm{mg} / \mathrm{dL}$ and mildly elevated amylase level around $200 \mathrm{U} / \mathrm{L}$.

An immediate angiography through the right femoral artery was performed where aortography showed normal anatomy without celiac stenosis. A selective cannulation of the superior mesenteric artery showed an aneurysm in the inferior pancreaticoduodenal artery (Figure 3 ). A highly selective 
cannulation of the aneurysmal branch, using a microcatheter, followed by an embolization using $3 \mathrm{~mm}$ coils (A Cook Group Company, Bloomington, IN, USA) was successfully performed (Figure 4).

Next day the patient maintained a normal level of hemoglobin, but blood amylase level was elevated above 2,000 U/L. After a few days of conservative treatment for pancreatitis which included 2 days of nil per os (NPO), intravenous fluids, and analgesics, the patient was discharged in very good condition 8 days after embolization, with a decreased level of amylase (181 U/L).

The patient was admitted again about 1 month after the index admission with pancreatitis and a retroperitoneal hematoma with no active bleeding, and was treated

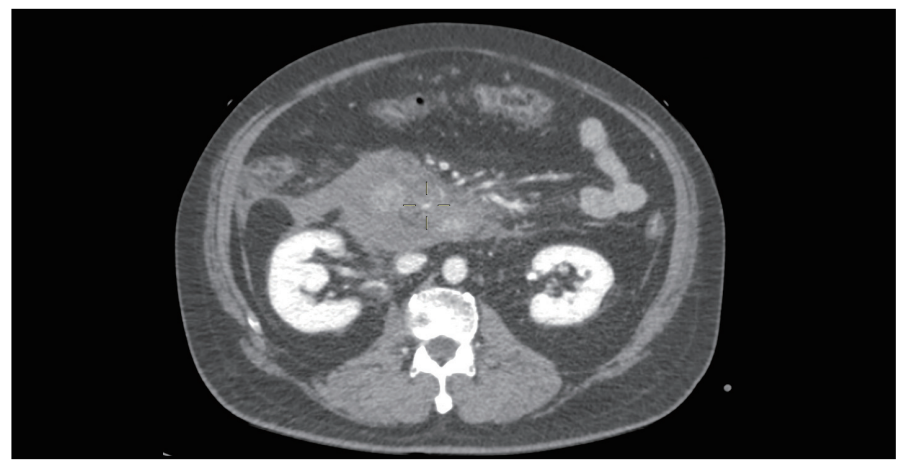

Figure 1. CT angiography showed a hematoma between the duodenum and the pancreas with an active extravasation of contrast.

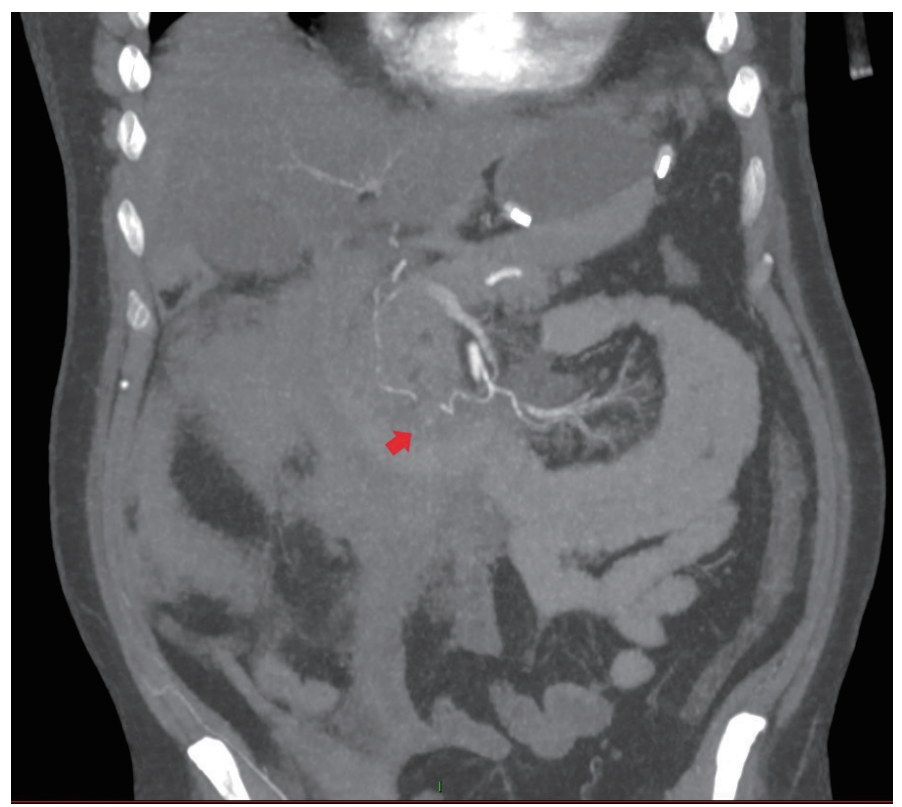

Figure 2. CT angiography showed a hematoma between the duodenum and the pancreas with an active extravasation of contrast (red arrow). conservatively. It's worth noting that during this admission, several causes of acute pancreatitis were ruled out including cholelithiasis, hypertriglyceridemia, and a history of alcohol or drug intake.

The patient was followed up in the outpatient clinic 2 weeks after discharge, and was making a good recovery.

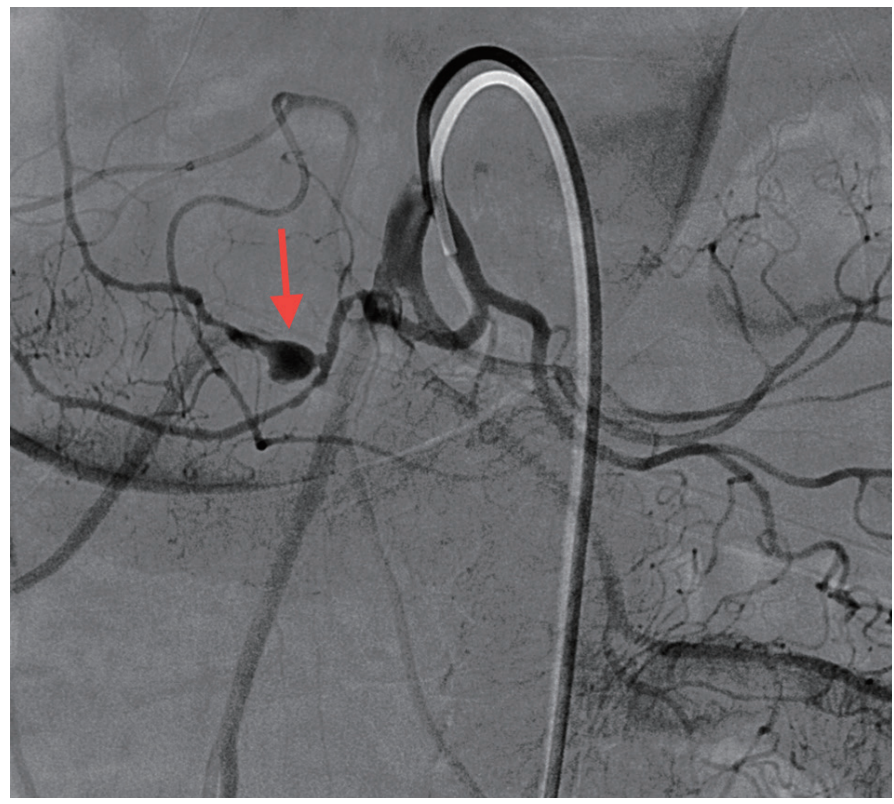

Figure 3. Angiography showed an aneurysm in the inferior pancreaticoduodenal artery (red arrow).

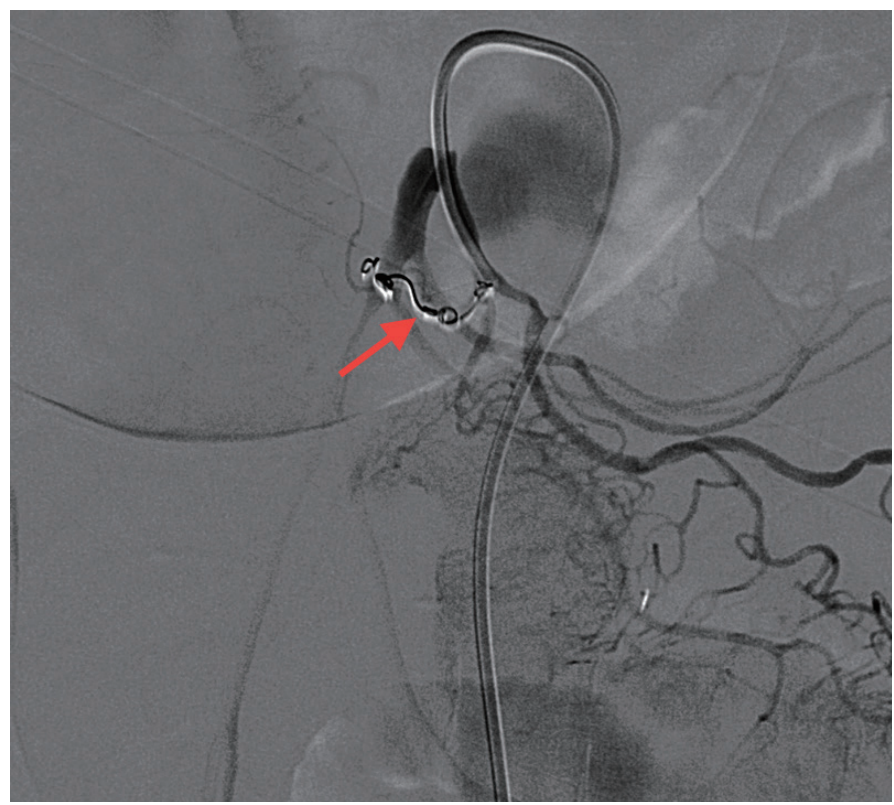

Figure 4. Angiographic coil embolization of the inferior pancreaticoduodenal artery (red arrow). 


\section{Discussion}

The occurrence of visceral artery aneurysms are rare, only appearing in $0.02 \%-0.1 \%$ of cases [5]. The prevalence of superior and inferior PDA aneurysms accounts for approximately $10 \%$ of all visceral artery aneurysms [4] .

Generally speaking, there are 2 types of PDA aneurysms; the more prevalent true aneurysms and the less common false aneurysms [3]. Although in the acute settings this is thought to be irrelevant, the patient in this case potentially had a true aneurysm, possibly caused by his previous history of smoking. In addition, no symptoms of pancreatitis were reported.

There are diversified clinical pictures of patients presenting with PDA aneurysm, with the majority of previously reported cases presenting signs of epigastric outlet obstruction including: epigastric pain, nausea and vomiting [6,7], while other cases presented with signs of gastrointestinal bleeding and anemia [8]. The patient in this study presented with an extremely unusual clinical picture of PDA aneurysm, with hemorrhagic shock due to intra-abdominal bleeding.

PDA aneurysms can be observed by a variety of imaging techniques including computed tomography, ultrasound, magnetic resonance imaging, and digital subtraction angiography. By using these techniques different characteristics of the aneurysms can be manifested including size, morphology, location, and associated surrounding arteries. Results from these scans in turn constitute a guide to further management. In this case an abdominal computed tomography angiography was performed which showed a hematoma with active bleeding in the head of the pancreas which may correspond to a bleeding from an underlying aneurysm or pseudoaneurysm in that region.

It is worth mentioning that previously reported cases of PDA aneurysms used different management strategies including surgical and non-surgical treatments. However, non-surgical strategies presented by endovascular treatment, are considered the first line treatment for PDA aneurysms. This technique is usually performed by coil embolization, which has a success rate of 72.7\% [9]. Coil embolization was successfully performed to treat the patient in this case study who was later discharged after making good progress in recovery.

Regarding the patient's elevated amylase levels in the post embolization period, it is believed that the high amylase level was caused by the ischemic effect of the embolization on the pancreatic tissue.

Although it's not a distinct cause of pancreatitis, a few cases of ischemic pancreatitis have been reported after embolization of gastroduodenal artery aneurysm [10].

Acute care surgeons and emergency physicians should be aware of this very rare case because if spontaneous intrabdominal bleeding is not diagnosed and treated appropriately, the outcome may be fatal. It's important for emergency physicians to keep in mind the rare causes of intrabdominal bleeding, and to be aware that invasive radiology is an important and lifesaving intervention for the treatment of visceral aneurysms and pseudoaneurysms.

\section{Conflicts of Interest}

The authors have no conflicts of interest to declare.

\section{References}

[1] Suzuki K, Tachi Y, Ito S, Maruyama K, Mori Y, Komada T, et al. Endovascular management of ruptured pancreaticoduodenal artery aneurysms associated with celiac axis stenosis. Cardiovasc Intervent Radiol 2008;31(6):1082-7.

[2] al-Jeroudi A, Belli AM, Shorvon PJ. False aneurysm of the pancreaticoduodenal artery complicating therapeutic endoscopic retrograde cholangiopancreatography. Br J Radiol 2001;74(880):375-7.

[3] Sutton D, Lawton G. Coeliac stenosis or occlusion with aneurysm of the collateral supply. Clin Radiol 1973;24(1):49-53.

[4] Williams M, Alderson D, Virjee J, Callaway M. CT-guided percutaneous thrombin injection for treatment of an inferior pancreaticoduodenal artery pseudoaneurysm. Cardiovasc Intervent Radiol 2006;29(4):669-71.

[5] Dallara H, Habboushe J. Spontaneous inferior pancreaticoduodenal artery pseudoaneurysm rupture. Intern Emerg Med 2017;12(8):1319-21.

[6] Alhasan A, Khan PM. An unusual cause of gastric outlet obstruction: pancreaticoduodenal artery aneurysm. Ann Saudi Med 2009;29(5):393-6.

[7] Androulakakis Z, Paspatis G, Hatzidakis A, Kokkinaki M, Papanicolaou N, Grammatikakis I, et al. Gastric outlet obstruction caused by a giant gastroduodenal artery aneurysm: a case report. Eur J Gastroenterol Hepatol 2001;13(1):59-61.

[8] Barbiero G, Battistel M, Susac A, Miotto D. Percutaneous thrombin embolization of a pancreatico-duodenal artery pseudoaneurysm after failing of the endovascular treatment. World J Radiol 2014;6(8):629-35.

[9] Ikeda O, Tamura Y, Nakasone Y, Iryou Y, Yamashita Y. Nonoperative management of unruptured visceral artery aneurysms: Treatment by transcatheter coil embolization. J Vasc Surg 2008;47(6):1212-9.

[10] Han SP, Galketiya K. Ischaemic pancreatitis and pseudocyst: a rare complication of embolization of gastroduodenal artery aneurysm. ANZ J Surg 2018;88(3):E204-5. 